\title{
Failure patterns of cervical lymph nodes in metastases of unknown origin according to target volume
}

\author{
Dong-Yun Kim², Dae Seog Heo², Bhumsuk Keam², Chan Young Ock², Soon Hyun Ahn , Ji-hoon Kim, \\ Kyeong Cheon Jung ${ }^{5}$, Jin Ho Kim ${ }^{1,6,7}$, Hong-Gyun $\mathrm{Wu}^{1,6,7}$ \\ ${ }^{1}$ Department of Radiation Oncology, Seoul National University College of Medicine, Seoul, Korea \\ ${ }^{2}$ Department of Internal Medicine, Seoul National University College of Medicine, Seoul, Korea \\ ${ }^{3}$ Department of Otorhinolaryngology, Seoul National University College of Medicine, Seoul, Korea \\ ${ }^{4}$ Department of Radiology, Seoul National University College of Medicine, Seoul, Korea \\ ${ }^{5}$ Department of Pathology, Seoul National University College of Medicine, Seoul, Korea \\ ${ }^{6}$ Cancer Research Institution, Seoul National University College of Medicine, Seoul, Korea \\ ${ }^{7}$ Institute of Radiation Medicine, Medical Research Center, Seoul National University, Seoul, Korea
}

Received: February 28, 2020

Revised: March 25, 2020

Accepted: March 26, 2020

Correspondence:

Hong-Gyun Wu,

Department of Radiation Oncology,

Seoul National University College of

Medicine, 101 Daehak-ro, Jongno-gu,

Seoul 03080, Korea.

Tel: $+82-2-2072-3177$

Fax: +82-2-765-3317

E-mail:wuhg@snu.ac.kr

ORCID:

https://orcid.org/0000-0002-0167-7991
Purpose: This study was aim to evaluate the patterns of failure according to radiotherapy (RT) target volume for cervical lymph nodes in metastases of unknown primary origin in head and neck region (HNMUO).

Materials and Methods: Sixty-two patients with HNMUO between 1998 and 2016 were retrospectively reviewed. We analyzed the clinical outcomes and primary site failure depending on the radiation target volume. The target volume was classified according to whether the potential head and neck mucosal sites were included and whether the neck node was treated involved side only or bilaterally.

Results: Potential mucosal site RT (mucosal RT) was done to 23 patients and 39 patients did not receive mucosal RT. Mucosal RT showed no significant effect on overall survival (OS) and locoregional recurrence (LRR). The location of primary site failure encountered during follow-up period was found to be unpredictable and $75 \%$ of patients with recurrence received successful salvage therapies. No significant differences in OS and LRR were found between patients treated to unilateral $(n=35)$ and bilateral neck irradiation $(n=21)$. Treatment of both necks resulted in significantly higher mucositis. Conclusions: We found no advantages in OS and LRR of patients with HNMUO when mucosal sites and bilateral neck node were included in the radiation target volume.

Keywords: Metastases of unknown primary origin in head and neck region, Pattern of failure, Radiotherapy

\section{Introduction}

Cervical lymph node in metastasis of unknown primary origin in head and neck region (HNMUO) is a heterogeneous group of malignancy involving the cervical lymph node metastases without obvious primary site despite comprehensive evaluations [1-4]. Squamous cell carcinoma is the most common histology in 70\% to $90 \%$ of patients diagnosed with HNMUO patients, followed by undiffer- entiated carcinoma and adenocarcinoma [5,6]. HNMUO accounts for $1.5 \%$ to $7 \%$ of all head and neck carcinoma [5,7-9]. Because of this low incidence, no randomized clinical trials have been undertaken and consensus has not been reached to establish the optimal treatment outcomes.

Treatment of HNMUO generally consists of combined modalities of surgery, radiation therapy (RT) and chemotherapy to maximize tumor control while reducing toxicity. Traditional approaches in- 
clude either neck dissection followed by adjuvant RT with or without chemotherapy, or primary chemotherapy/chemoradiotherapy followed by neck dissection for the management of more advanced disease $[4,7,8,10-12]$. However, the optimal target volume for RT is controversial, with wide variations in RT of the involved neck alone and potential head and neck mucosal sites. Elective treatment of the bilateral neck is also an important issue considering the target volume to minimize the side effects.

The goal of the present study is to compare the clinical outcomes according to the target volume whether or not including potential mucosal sites and bilateral neck, and to identify the patterns of failure to suggest the optimal radiation field for HNMUO patients.

\section{Materials and Methods}

\section{Patient population and diagnostic workup}

After the approval of the Institutional Review Board of Seoul National University Hospital (No. H-1803-132-932), a retrospective review of 69 patients with HNMUO between 1998 and 2016, was performed. The patients underwent treatment with curative intent including radiation therapy. Sixty-two patients were eligible for inclusion in this study, except for patients who had metastatic carcinoma in the solely supraclavicular node $(n=6)$ or prior head and neck malignancy $(n=1)$. All patients had good performance status scored by the Eastern Cooperative Group (ECOG) performance status 0 to 2. Patients who received incomplete RT or who failed to follow-up continuously were excluded from the analyses.

All 62 patients manifested histologically proven metastatic carcinoma in cervical lymph nodes, and 56.6\% of them had undergone directed biopsies from suspicious primary sites such as tonsil, nasopharynx, and the base of tongue. All the results of biopsies were reviewed by institutional pathologists.

The typical diagnostic workup of HNMUO patients consisted of clinical examination and endoscopy (i.e., nasopharyngoscopy and laryngoscopy), chest X-ray, complete blood count, liver function test, computerized tomography (CT) and/or magnetic resonance imaging (MRI) of the head and neck region. Especially, 88.7\% underwent positron emission tomography- computerized tomography (PET-CT). We restaged all patients retrospectively based on the criteria of the 8th edition of the American Joint Committee on Cancer staging system.

\section{Treatment modalities}

Of all 62 patients, 42 patients underwent primary neck dissection; 30 patients with modified radical neck dissection (MRND), 8 with radical neck dissection (RND), and 4 with selective neck dissection.

All patients received RT with curative intent. The 42 patients who underwent initial neck dissection surgeries were treated with postoperative radiotherapy, and the remaining patients underwent radical RT after biopsies. Most of the patients received either intensity-modulated radiotherapy (IMRT, 50.0\%) or three-dimensional conformal radiotherapy (3D-CRT, 40.3\%). Since we included patients who had been treated since 1998, 9.7\% of patients who received 2D-technique RT were also analyzed. Doses typically consisted of 70 Gy prescribed for gross disease, 60-63 Gy prescribed for high-risk or postoperative areas, 54-56 Gy for treatment of intermediate-risk subclinical regions, and 42-47.6 Gy to treat lower-risk areas.

Twenty-three patients (37.1\%) had RT to potential primary mucosal sites. Among them, 16 patients received RT to potential primary mucosa which had been biopsied during initial diagnostic work-up, even though negative result had been confirmed. The remaining 7 patients did not undergo mucosal biopsies at the time of initial diagnosis, but at the radiation oncologists' decision, 5 patients received RT to oropharynx, 1 to oropharynx and nasopharynx, and 1 to oropharynx and base of tongue. Most common site for potential primary mucosal RT was oropharynx (45.2\%), followed by nasopharynx (32.3\%), base of tongue (12.9\%).

In terms of chemotherapy, $30.6 \%$ of patients $(n=19)$ were offered neoadjuvant chemotherapy before RT. The regimens of induction chemotherapy included "5-fluorouracil (5-FU) and cisplatin" (n = 7), "docetaxel and cisplatin" ( $n=6)$, "docetaxel, cisplatin and 5-FU" ( $n=3$ ), and "paclitaxel and cisplatin" ( $n=3$ ). Half of the patients ( $n=31$ ) underwent concurrent chemotherapy during RT, and most of them were cisplatin-based regimens. Cisplatin alone was used to treat 29 patients, "5-FU, cisplatin, and cetuximab" was used in one patient each. The details of overall treatment were described in Fig. 1.

\section{Follow-up}

After therapy, patients were monitored during the first 2 weeks after RT and every 1 to 2 months later, then every 2 to 3 months during the first year of follow-up, every 4 months during the second, and every 6 months during the third year of follow-up. The follow-up interval was increased or decreased according to the patient's clinical evaluation.

\section{Statistical analyses}

We compared the overall survival (OS), locoregional recurrence $(\mathrm{LRR})$ and primary site failure depending on the radiation target volume. LRR was defined as any relapse in the ipsilateral neck node either in-field or out-field, contralateral neck node and/or head and neck mucosa. The target volume was classified according to whether the potential primary head and neck mucosal sites were 


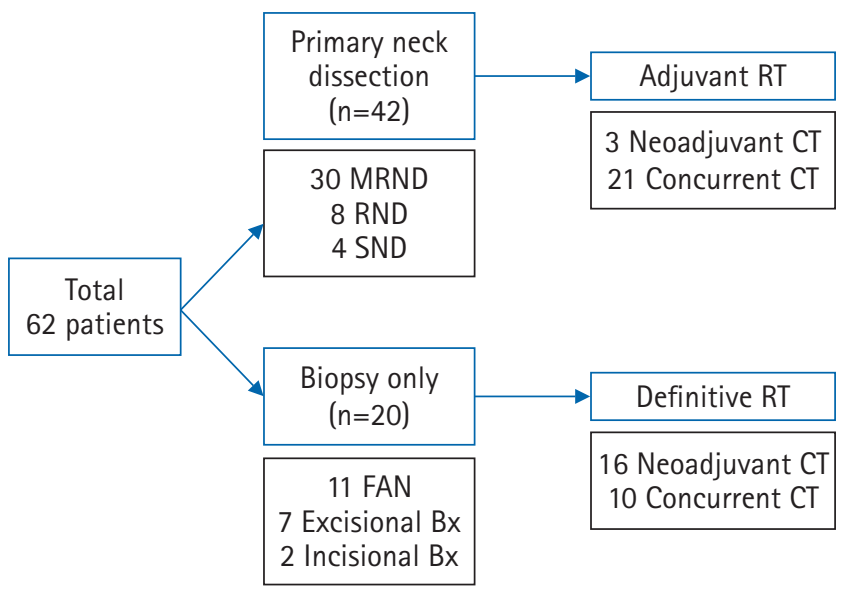

Fig. 1. Flow chart of overall treatment. MRND, modified radical neck dissection; RND, radical neck dissection; SND, selective neck dissection; FND, fine-needle aspiration; $\mathrm{Bx}$, biopsy; RT, radiotherapy; $\mathrm{CT}$, chemotherapy.

included and whether the neck node was treated unilaterally or bilaterally. Analyses of the clinical outcomes of neck node RT were performed except in patients who initially presented metastatic lymph nodes in the bilateral neck. All analyses were performed using Stata/MP 15.0 (StataCorp., College Station, TX, USA) with a significance level of 0.05 .

\section{Results}

\section{Patient characteristics}

The median follow-up time was 44.1 months (range, 5.5 to 149.0 months). Sixty-two patients met the inclusion criteria. The mean age of the patients was 58.1 years (range, 20 to 77 years), including 55 males and 7 females. All patients had good performance status defined by ECOG performance status score of 0 or 1 , except for 1 patient. The number of patients with a history of smoking was $26(41.9 \%)$.

The most common type of histology was squamous cell carcinoma in $83.9 \%$, followed by poorly differentiated carcinoma in 16.1\%. The N stages classified as N1, N2a, N2b, N2c, N3a, and N3b were $2,16,26,6,6$, and 6 patients, respectively. Eleven patients manifested extra-nodal extensions. Among them, 1 patient was diagnosed with N2a, 3 patients were N2b, and 1 patient showed N3a disease. Besides, there was 1 patient with N3a and 5 patients were confirmed as N3b stage. Histologic staining and immunohistochemistry of neck nodes for human papillomavirus (HPV) and Epstein-Barr virus (EBV) were reviewed, but only $40.3 \%$ and $25.8 \%$ of patients were tested for HPV and EBV, respectively. Of all patients, 17.7\% for HPV and 3.2\% for EBV tested positive.

Forty-two patients (67.7\%) underwent primary neck dissection before RT, 11 patients received RT after fine-needle aspiration (FNA) only, and 9 patients underwent either excisional $(n=7)$ or an incisional $(n=2)$ biopsies. Primary neck dissection was not performed at the clinician's discretion in patient with initial bulky disease or old age, and when the patient did not want surgery.

Most of the patients (88.7\%) were exposed to PET-CT for the initial diagnoses to rule out a primary tumor site and $74.2 \%$ of patients underwent biopsy for suspected primary mucosal sites, including tonsil, tongue base and nasopharynx. The most common site for mucosal biopsy was tonsil, and biopsies at multiple sites were performed in each patient depending on the otolaryngologist's judgment. Treatments to putative head and neck mucosal sites and bilateral neck nodes depended on the radiation oncologist's discretion. Twenty-three patients were treated with RT for suspected mucosal sites even though site-directed biopsies were negative, if the clinical judgment indicated a strong suspicion.

\section{Mucosal RT}

Potential primary mucosal RT was administered to the $37.1 \%$ ( $n=$ 23) of patients whereas $62.9 \%(n=39)$ were not exposed to mucosal RT. There was a statistical difference in the rate of neoadjuvant chemotherapy and the extent of neck node irradiation between the two treatment groups. Most patients who received mucosal RT did not undergo neoadjuvant chemotherapy (91.7\%) and were treated for electively bilateral neck nodes together (73.9\%) (Table 1).

Potential primary mucosal RT showed no significant effect on overall survival $(p=0.376)$. The 5 -year OS rate was $61.8 \%$ in the mucosal RT (+) group and 53.9\% in the mucosal RT (-) group (Fig. 2). Also, there was no statistically significant difference in the LRR between the groups depending on the mucosal RT ( $p=0.099)$. The 5 -year LRR rate was $10.7 \%$ in mucosal RT (+) and $30.2 \%$ in mucosal RT (-) group. No differences were found in the ipsilateral infield, ipsilateral out-field failure, and contralateral failure rate between the two treatments. Though mucosal RT showed trend for lower primary site failure $(p=0.087)$, only 4 patients $(10.3 \%)$ who were with untreated potential primary site developed subsequent mucosal head and neck carcinoma. Moreover, the location of primary site failure was found to be unpredictable, which included 1 case of nasopharynx, 1 involving oropharynx, and 2 involving oral cavities (buccal and gingiva). These primary site failures did not match the lesions predicted by biopsy and imaging etc. in the initial workup. One patient showed mucosal recurrence at the base of tongue after mucosal irradiation to nasopharynx. Among the mucosal RT (-) patients, 75\% patients $(n=3)$ who showed recurrence as the primary site failure underwent successful salvage surgery followed by adjuvant chemotherapy. One patient who showed re- 
Table 1. Characteristics of patients treated with mucosal RT (+) vs. mucosal RT (-)

\begin{tabular}{|c|c|c|c|}
\hline & Mucosal RT $(+)(n=23)$ & Mucosal RT $(-)(n=39)$ & p-value \\
\hline Age at diagnosis (yr) & $54.7 \pm 10.1(20-72)$ & $60.1 \pm 9.6(36-77)$ & $0.023^{\text {a) }}$ \\
\hline Sex & & & $0.185^{b)}$ \\
\hline Male & $22(95.7)$ & $33(84.6)$ & \\
\hline Female & $1(4.4)$ & 6 (15.4) & \\
\hline History of smoking & & & $0.731^{\mathrm{b})}$ \\
\hline Yes & $9(39.1)$ & $17(43.6)$ & \\
\hline ECOG performance status & & & $0.439^{b)}$ \\
\hline $0-1$ & $23(100)$ & $98(97.4)$ & \\
\hline$\geq 2$ & $0(0)$ & $1(2.6)$ & \\
\hline N-classification (AJCC 8th) & & & $0.120^{\mathrm{b})}$ \\
\hline N1 & $0(0)$ & $2(5.1)$ & \\
\hline N2 & $21(91.3)$ & $27(69.2)$ & \\
\hline N3 & $2(8.3)$ & $10(25.6)$ & \\
\hline Pathologic features & & & $0.503^{b)}$ \\
\hline $\mathrm{SqCC}$ & 18 (78.3) & $34(85.2)$ & \\
\hline Poorly differentiated & $5(21.7)$ & $5(12.8)$ & \\
\hline \multicolumn{4}{|l|}{ FDG-PET at diagnosis } \\
\hline Taken & $21(91.3)$ & 34 (87.2) & $0.620^{b)}$ \\
\hline \multicolumn{4}{|l|}{ Neoadjuvant chemotherapy } \\
\hline Yes & $2(8.3)$ & $17(43.6)$ & $0.004^{b)}$ \\
\hline \multicolumn{4}{|l|}{ Concurrent chemotherapy } \\
\hline Yes & $10(41.7)$ & $21(53.9)$ & $0.430^{b)}$ \\
\hline \multicolumn{4}{|l|}{ RT field } \\
\hline Unilateral & $6(26.1)$ & $29(74.4)$ & $0.001^{b)}$ \\
\hline Bilateral & 17 (73.9) & $10(25.6)$ & \\
\hline
\end{tabular}

Values are presented as mean \pm standard deviation (range) or number (\%).

RT, radiotherapy; ECOG, Eastern Cooperative Oncology Group; AJCC, American Joint Committee on Cancer; SqCC, squamous cell carcinoma; FDG-PET, fluorodeoxyglucose-positron emission tomography.

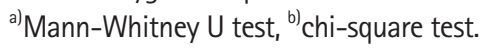

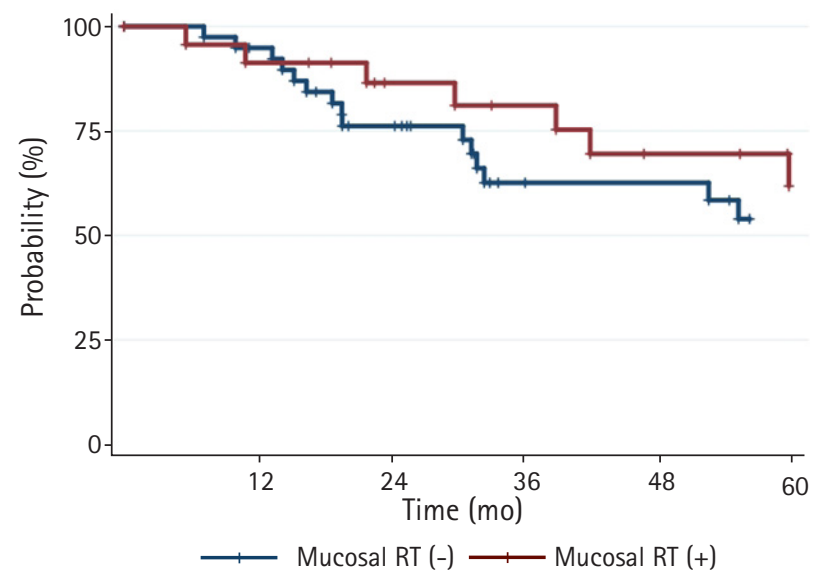

Fig. 2. Actuarial overall survival curves of patients receiving potential primary mucosal RT (+) vs. mucosal RT (-) ( $p=0.376$ by log-rank test). RT, radiotherapy. currence as systemic progression failed to undergo salvage therapy, and instead received palliative chemotherapy (Table 2). In toxicity analyses, irradiation to potential primary mucosa did not result in any differences in the incidence of mucositis ( $p=0.412)$, dermatitis ( $p=0.126)$, unexpected hospitalization $(p=0.276)$ or weight loss greater than $10 \%(p=0.980)$.

\section{Unilateral vs. bilateral neck irradiation}

After excluding initially bilateral neck diseases ( $n=6), 35$ patients received involved unilateral neck RT only and 21 underwent elective bilateral neck RT. There was tendency of involved unilateral neck RT for patients who had taken PET-CT at initial work-up ( $p=$ 0.017). Among the patients who received bilateral neck RT, $66.7 \%$ received potential primary mucosal RT concurrently $(p=0.001)$ (Table 3).

Compared with the unilateral neck RT, the bilateral neck RT did not improve the OS ( $p=0.403)$. The actuarial 5-year OS rate was 
Table 2. Patterns of failure according to mucosal RT, and unilateral vs. bilateral neck irradiation

\begin{tabular}{lcccc}
\hline & Mucosal RT $(+)(n=23)$ & Mucosal RT $(-)(n=39)$ & Unilateral irradiation $(n=35)$ & Bilateral irradiation $(n=21)$ \\
\hline $\begin{array}{l}\text { All locoregional failure } \\
\text { Regional failure }\end{array}$ & 3 & 10 & & 4 \\
$\quad$ Ipsilateral in-field failure & 2 & 5 & 4 & 2 \\
$\quad$ Ipsilateral out-field failure & 1 & 2 & 2 & 0 \\
$\quad$ Contralateral failure & 0 & 1 & 0 & 1 \\
Local failure (mucosa) & 1 & 4 & 10 & 2 \\
Distant failure & 8 & 11 & 2 & 6 \\
\hline
\end{tabular}

Table 3. Characteristics of patients treated with unilateral vs. bilateral neck irradiation

\begin{tabular}{|c|c|c|c|}
\hline & Unilateral RT $(n=35)$ & Bilateral RT $(n=21)$ & $\mathrm{p}$-value \\
\hline Age at diagnosis (yr) & $60.4 \pm 9.0(36-77)$ & $54.6 \pm 12.1(20-72)$ & $0.065^{a)}$ \\
\hline Sex & & & $0.175^{b)}$ \\
\hline Male & $29(82.9)$ & $20(95.2)$ & \\
\hline Female & $6(17.1)$ & $1(4.8)$ & \\
\hline History of smoking & & & $0.480^{b)}$ \\
\hline Yes & $15(42.9)$ & 7 (33.3) & \\
\hline ECOG performance status & & & $0.434^{b)}$ \\
\hline $0-1$ & $34(97.1)$ & $21(100)$ & \\
\hline$\geq 2$ & $1(2.9)$ & $0(0)$ & \\
\hline N-classification (AJCC 8th) & & & $0.436^{b)}$ \\
\hline N1 & $2(5.7)$ & $0(0)$ & \\
\hline N2 & $26(74.3)$ & 18 (85.7) & \\
\hline N3 & $7(20.0)$ & $3(14.3)$ & \\
\hline \multicolumn{4}{|l|}{ Pathologic features } \\
\hline $\mathrm{SqCC}$ & 29 (82.9) & $17(80.9)$ & $0.414^{b)}$ \\
\hline Poorly differentiated & $6(17.1)$ & $4(19.1)$ & \\
\hline \multicolumn{4}{|l|}{ FDG-PET at diagnosis } \\
\hline Taken & $34(97.1)$ & $15(71.4)$ & $0.005^{b)}$ \\
\hline \multicolumn{4}{|l|}{ Neoadjuvant chemotherapy } \\
\hline Yes & $10(27.8)$ & $6(28.6)$ & $1.000^{b)}$ \\
\hline \multicolumn{4}{|l|}{ Concurrent chemotherapy } \\
\hline Yes & $19(54.3)$ & $8(38.1)$ & $0.240^{b)}$ \\
\hline \multicolumn{4}{|l|}{ Suspected mucosal RT } \\
\hline Yes & $6(17.1)$ & $14(66.7)$ & $0.001^{b)}$ \\
\hline
\end{tabular}

Values are presented as mean \pm standard deviation (range) or number (\%).

RT, radiotherapy; ECOG, Eastern Cooperative Oncology Group; AJCC, American Joint Committee on Cancer; SqCC, squamous cell carcinoma; FDG-PET, fluorodeoxyglucose-positron emission tomography.

${ }^{a}$ Mann-Whitney $\mathrm{U}$ test, ${ }^{\text {b) }}$ chi-square test.

$55.9 \%$ in the unilateral RT and 67.9\% in the bilateral RT group (Fig. 3). Also, there was no significant difference in LRR between the two fields ( $p=0.378$ ). The 5 -year LRR rate was $26.0 \%$ in the unilateral and $12.3 \%$ in the bilateral RT group. There were 2 patients with primary mucosa failure in both unilateral and bilateral neck RT groups. When analyzing the patterns of failure, there were 6 patients who showed regional relapses in the unilateral RT group. They included four patients with ipsilateral in-field failures, which resulted in recurrences in previously irradiated areas. There were 2 patients who showed ipsilateral out-field failures (Table 2). Those 2 patients initially underwent cervical lymphadenopathy in the left level II and left level II/III, followed by unilateral neck RT including left level II, III, IV, Va, and Vb. After the treatment, 2 patients showed recurrence at left level la or lb. Elective bilateral RT resulted in recurrences at ipsilateral in-field in 2 patients and even recurrence at the contralateral neck in 1 patient. In addition, when 


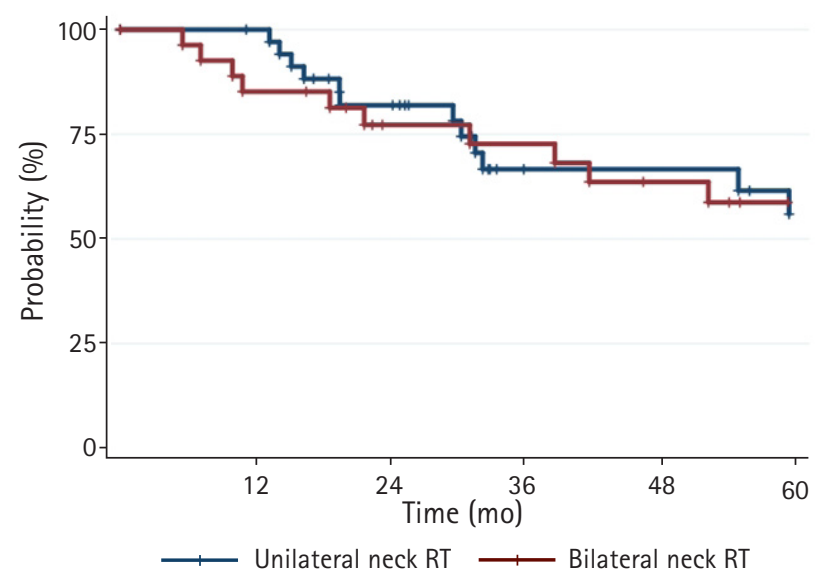

Fig. 3. Actuarial overall survival curves of patients treated with involved unilateral vs. elective bilateral neck RT ( $p=0.403$ by log-rank test). RT, radiotherapy.

treated with RT for both necks, grade 2+ mucositis occurred significantly ( $p=0.047)$.

\section{Discussion and Conclusion}

Currently, there is no consensus for the optimal treatment of HNMUO. The present study was conducted to analyze the clinical outcomes and patterns of failure in patients with HNMUO according to the RT target volume, and suggest RT field to improve tumor control rates with minimal toxicity.

According to the latest National Comprehensive Cancer Network (NCCN) guideline for the management of occult primary head and neck carcinoma, the RT field should be based on the initial tumor size, nodal station, and HPV and EBV status. However, in light of specific principles of RT, no guidelines are available whether the putative mucosa as primary site should be included even though PET-CT revealed negative, and whether only involved side of neck should be treated or not.

Despite the lack of prospective trials, multiple retrospective studies have shown conflicting results regarding the RT fields. Several previous studies showed that comprehensive RT including suspicious mucosa and bilateral neck field were beneficial in reducing the failure rates $[2,13,14]$. A recent study by Kamal et al. [4], reported that comprehensive IMRT to both neck, oropharyngeal and nasopharyngeal mucosa resulted in statistically higher rates of OS and disease control. However, this study did not indicate the number of patients exposed to PET-CT during the initial diagnosis. Currently, it is common practice to undergo PET-CT at initial assessments, and the probability of finding an occult primary site would be $20 \%-25 \%$. Therefore, it is necessary to consider when the pa- tient was treated, when PET-CT was not routinely used in the initial diagnosis. However, a few studies in the past revealed no differences in OS, relapse-free survival, and the emergence of primary site between comprehensive and limited RT fields [15-17]. A recent review article by the GEORCC (Grupo de Tumores de Cabeza y Cuello) recommended clinical target volume (CTV) in clinical practice for contouring HNMUO. First, regarding mucosal CTV, potential mucosal site RT might be possible in case of $\mathrm{N} 1$ with extranodal extension or N2/N3 disease. The findings suggested that patients receive mucosal irradiation on carefully considering HPV/p16 and EBV status. As for the nodal CTV, patients diagnosed as N1-N2A and pN2B with $\geq 2$ affected lymph nodes (LNs) could be treated with involved neck RT alone. However, elective bilateral neck RT would be beneficial in the case of N2B with > 2 positive LNs or N2C-N3 patients. However, no consensus has been set regarding the optimal RT for HNMUO [12].

In the present study, we analyzed the results of clinical outcomes involving HNMUO patients classified according to whether the RT field included the potential primary mucosa or involved neck RT only (vs. elective bilateral neck RT). In comparison with other published studies, a high proportion (88.7\%) of patients underwent PET-CT during the initial staging. Our study found that potential primary mucosal RT and bilateral neck irradiation yielded no clinical benefits in OS and LRR. Based on the analysis of patterns of failure, the location of subsequent primary failure was unpredictable. Even after bilateral neck RT, 2 patients showed recurrence at the ipsilateral in-field area and another patient showed contralateral in-field failure. Two patients treated with involved neck RT only showed recurrence in the ipsilateral out-field area. Two patients who initially manifested lymphadenopathy at left level II and II/III received unilateral RT to left level II-V, and showed recurrence at left la/lb. Even elective bilateral neck RT showed significantly greater mucositis. Based on these results, omission of mucosal RT and bilateral neck irradiation might be an option to maintain treatment efficacy while minimizing RT-related toxicities. Because salvage therapies could be effectively performed except systemic progression, limiting the extent of RT may be helpful in the salvage surgery at the time of recurrence. On the other hand, it was noteworthy that when mucosal RT was not performed, the 5-year LRR was $30.2 \%$, which was higher than $10.7 \%$ of receiving mucosal RT. Although it was not statistically significant and the location of subsequent mucosal primary was also unpredictable, validation in the study with a larger number of patients would be necessary.

This study had several limitations. It had inherent weak points due to the retrospective nature of the study. The number of analyzed patients was insufficient to confirm the results. In addition, it was not clear how the radiation oncologist treated HNMUO pa- 
tients, especially on what criteria the potential primary mucosa and elective bilateral neck nodes were treated. In addition, of all patients, 74.2\% for EBV and 59.7\% for HPV were not tested. As the viral tests for EBV and HPV are common practice for determining coverage of nasopharynx and/or oropharynx in the RT field nowadays $[4,8,9]$, the lack of data regarding the viral tests might be major limitation in interpreting the results. It was also found that 5 -year LRR in this study was rather higher (10.7\% vs. 5.8\%) compared to other study by Wang et al. [18] published in recent year.

Nevertheless, the results obtained from this study might be meaningful in the actual clinical setting, where the incidence of HNMUO is very rare and the treatment guidelines are scarce. To date, no clear treatment consensus is available, and therefore, physicians may have different judgment criteria for optimal therapies even in the same institution. In particular, opinions on RT field, such as treating potential primary mucosa or involved neck only, can vary among radiation oncologists. Since the criteria for treating HNMUO were different among individual radiation oncologists even in a single institution, it was difficult to establish the rules for RT fields. However, just as the prevalence of disease is very low, the lack of a standard of care is also an inevitable problem of HNMUO itself. The absence of HPV/EBV staining in many patients was a major flaw of this paper. This was unavoidable because the patients were distributed over a long period of 18 years from 1998 to 2016, and the viral staining for HPV/EBV has only recently been done routinely. In addition, the reason why the LRR of this study was higher than that of Wang et al. [18] was that the initial N2/3 stage was $95.2 \%$ in our study, but only $49.4 \%$ in Wang's paper. Despite several inevitable shortcomings, the advantage of this study was high rates of PET-CT were taken at the time of initial evaluation. The high percentage of PET-CT indicated the accuracy of the diagnosis, and it could be assumed that the initial PET-CT had largely eliminated the probability of finding a hidden primary site.

In conclusion, we found that there were no benefits in OS and LRR for patients diagnosed with HNMUO when treating head and neck mucosal sites and bilateral neck nodes in the radiation target filed. The primary site failure was hard to predict and salvage therapy was successfully performed in case of subsequent mucosal carcinoma.

\section{Conflict of Interest}

No potential conflict of interest relevant to this article was reported.

\section{Acknowledgements}

This study was supported by the National R\&D Program for Cancer Control, Ministry of Health and Welfare, Republic of Korea (No.1631200).

\section{References}

1. Mendenhall WM, Mancuso AA, Parsons JT, Stringer SP, Cassisi NJ. Diagnostic evaluation of squamous cell carcinoma metastatic to cervical lymph nodes from an unknown head and neck primary site. Head Neck 1998;20:739-44.

2. Klem ML, Mechalakos JG, Wolden SL, et al. Intensity-modulated radiotherapy for head and neck cancer of unknown primary: toxicity and preliminary efficacy. Int J Radiat Oncol Biol Phys 2008;70:1100-7.

3. Reddy SP, Marks JE. Metastatic carcinoma in the cervical lymph nodes from an unknown primary site: results of bilateral neck plus mucosal irradiation vs. ipsilateral neck irradiation. Int J Radiat Oncol Biol Phys 1997;37:797-802.

4. Kamal M, Mohamed AS, Fuller CD, et al. Outcomes of patients diagnosed with carcinoma metastatic to the neck from an unknown primary source and treated with intensity-modulated radiation therapy. Cancer 2018;124:1415-27.

5. Al Kadah B, Papaspyrou G, Linxweiler M, et al. Cancer of unknown primary (CUP) of the head and neck: retrospective analysis of 81 patients. Eur Arch Otorhinolaryngol 2017;274:2557-66.

6. Shoushtari A, Saylor D, Kerr KL, et al. Outcomes of patients with head-and-neck cancer of unknown primary origin treated with intensity-modulated radiotherapy. Int J Radiat Oncol Biol Phys $2011 ; 81: e 83-91$.

7. Studer G, Huber GF, Holz E, Glanzmann C. Less may be more: nodal treatment in neck positive head neck cancer patients. Eur Arch Otorhinolaryngol 2016;273:1549-56.

8. Muller von der Grun J, Tahtali A, Ghanaati S, Rodel C, Balermpas P. Diagnostic and treatment modalities for patients with cervical lymph node metastases of unknown primary site: current status and challenges. Radiat Oncol 2017;12:82.

9. Cuaron J, Rao S, Wolden S, et al. Patterns of failure in patients with head and neck carcinoma of unknown primary treated with radiation therapy. Head Neck 2016;38 Suppl 1:E426-31.

10. Beldi D, Jereczek-Fossa BA, D'Onofrio $A$, et al. Role of radiotherapy in the treatment of cervical lymph node metastases from an unknown primary site: retrospective analysis of 113 patients. Int J Radiat Oncol Biol Phys 2007;69:1051-8.

11. Perkins SM, Spencer CR, Chernock RD, et al. Radiotherapeutic management of cervical lymph node metastases from an un- 
known primary site. Arch Otolaryngol Head Neck Surg 2012; 138:656-61.

12. Cabrera Rodriguez J, Cacicedo J, Giralt J, et al. GEORCC recommendations on target volumes in radiotherapy for Head Neck Cancer of Unkown Primary. Crit Rev Oncol Hematol 2018; 130:51-9.

13. Grau $C$, Johansen LV, Jakobsen J, Geertsen $P$, Andersen $E_{\text {, J Jensen }}$ BB. Cervical lymph node metastases from unknown primary tumours: results from a national survey by the Danish Society for Head and Neck Oncology. Radiother Oncol 2000;55:121-9.

14. Nieder C, Gregoire V, Ang KK. Cervical lymph node metastases from occult squamous cell carcinoma: cut down a tree to get an apple? Int J Radiat Oncol Biol Phys 2001;50:727-33.

15. Weir $L$, Keane T, Cummings $B$, et al. Radiation treatment of cervi- cal lymph node metastases from an unknown primary: an analysis of outcome by treatment volume and other prognostic factors. Radiother Oncol 1995;35:206-11.

16. Sinnathamby K, Peters $\sqcup$, Laidlaw $C$, Hughes PG. The occult head and neck primary: to treat or not to treat? Clin Oncol (R Coll Radiol) 1997:9:322-9.

17. Marcial-Vega VA, Cardenes $H_{1}$ Perez $C A$, et al. Cervical metastases from unknown primaries: radiotherapeutic management and appearance of subsequent primaries. Int J Radiat Oncol Biol Phys 1990;19:919-28.

18. Wang Y, He SS, Bao Y, et al. Cervical lymph node carcinoma metastasis from unknown primary site: a retrospective analysis of 154 patients. Cancer Med 2018;7:1852-9. 